\title{
Aggressive Angiomyxoma: Only Time Will Tell
}

\author{
Aggressive Angiomyxoma: Only Time Will Tell
}

Dept. Of Radiodiagnosis \& Imaging, SKIMS, Soura, Srinagar, Jammu and Kashmir, India

\section{Article Info}

\section{Article Notes}

Received: August 30, 2018

Accepted: December 17, 2018

\section{*Correspondence:}

Dr. Saika Amreen, Dept. Of Radiodiagnosis \& Imaging, SKIMS, Soura, Srinagar, Jammu and Kashmir, India;

Telephone no: +919596257011

Email: saikaamreen@gmail.com

(C) 2018 Amreen S. This article is distributed under the terms of the Creative Commons Attribution 4.0 International License.

\section{ABSTRACT}

A 38-year-old primigravida delivered a healthy baby via cesarean section. The patient complained of vague abdominal discomfort a few weeks after surgery. Though blood work was unremarkable, an ultrasound revealed a deep pelvic hypoechoic collection. Patient was afebrile and blood work was unremarkable for infectious etiology. Hemoglobin was normal. The patient was otherwise healthy. This was 6 years ago. The lesion still persists.

\section{Case report}

At the initial presentation post-surgery, an ultrasound was performed which revealed a hypoechoic collection deep in pelvis in left inferolateral relation to the urinary bladder. A provisional diagnosis of post-surgical collection was made. However, patient refused any surgical intervention and was put on antibiotics. Few weeks later patient returned for follow up and repeat ultrasound revealed the same hypoechoic collection with similar measurements. Patient was still stable with unremarkable blood work. An MRI was performed with revealed a well-defined lesion in left lateral relation to the bladder. The lesion was isointense to muscle on T1W images. On T2W images the lesion was hyperintense with a swirling appearance. The lesion was seen to extend to the pelvic floor with infiltration into the ipsilateral levator muscle and subcutaneous fat with a part also extending to base of vulva. FNAC revealed only blood. A diagnosis of aggressive angiomyxoma was made. Patient

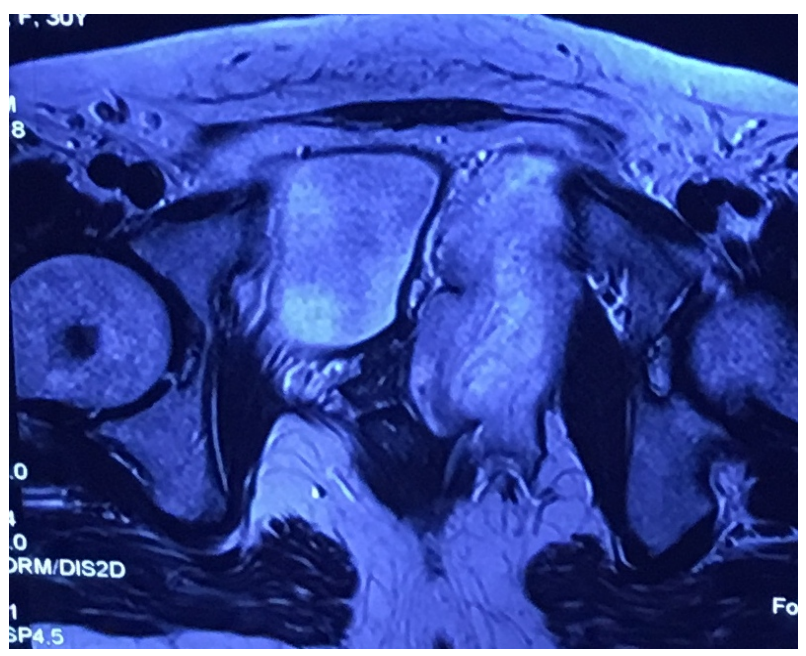

Figure 1: T2W axial image at level of femoral head revealing a hyperintense lesion in left lateral relation to the bladder with internal "swirling" architecture. The lesion is seen to extend beyond the pelvic floor and into subcutaneous planes on left side. These images have been taken 6 years ago. 


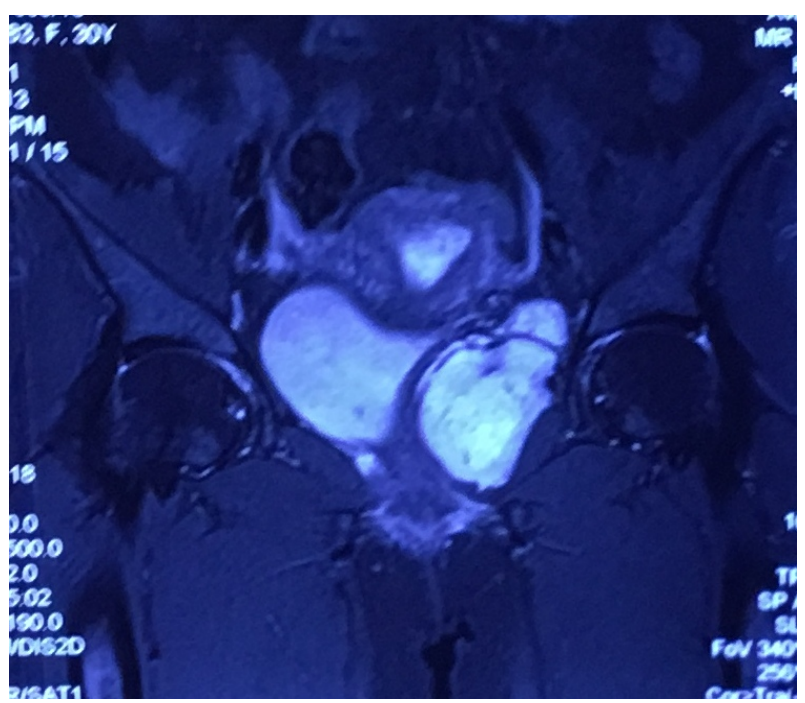

Figure 2: $\mathrm{T} 2 \mathrm{~W}$ coronal image showing relationship of the lesion with the postpartum uterus. The lesion is hyperintense with "swirling" appearance. Image taken 6 years ago.

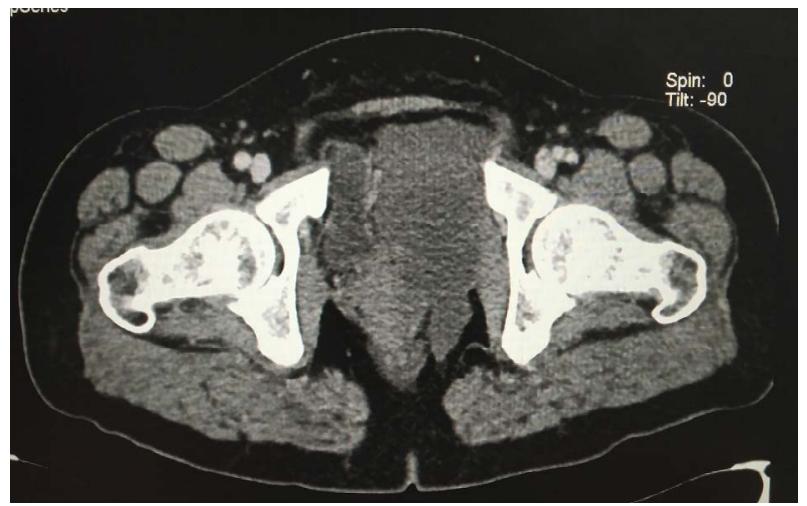

Figure 3: Axial contrast enhanced CT revealing a hypodense, poorly enhancing lesion in left lateral relation to bladder with extension into subcutaneous planes.

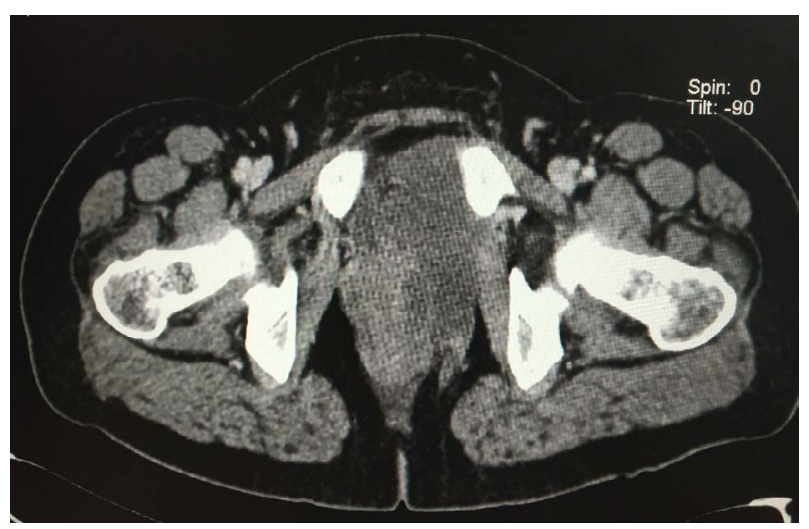

Figure 4: Axial contrast enhanced CT at few levels inferior to above revealing extension of the lesion into the levator muscles and subcutaneous tissue.

refused any further surgical intervention and was kept on follow up. Now 6 years after the initial diagnosis a follow up contrast CT was performed which revealed a hypodense lesion in left lateral relation to bladder with similar extensions. The lesion showed subtle post contrast enhancement. No definite interval growth was appreciated. No distant metastases found. A repeat ultrasound guided FNAC was performed which was found to have features of an angiomyxoid lesion. The patient refused any further intervention and is on follow up.

\section{Discussion}

The differential diagnosis of a solitary soft tissue perineal mass includes high-grade sarcoma, angiosarcoma, unclassified mesenchymal neoplasm, solitary fibrous tumor, superficial angiomyxoma and angiomyofibroblastoma ${ }^{1,2}$. This patient had a lesion that showed the distinctive imaging appearance of aggressive angiomyxoma - very high signal intensity on T2-weighted images and a swirled or layered internal architecture. ${ }^{3}$

Aggressive angiomyxomas are rare mesenchymal tumors that principally affect women of reproductive age with a peak incidence in the fourth to fifth decades of life; presenting as a painless mass or by local pressure effects. ${ }^{1}$ The name may be a misnomer because the tumor does not commonly display the clinical features of aggressive neoplasms, for example, rectal, vaginal, urethral, or vascular obstruction regardless of its intimate relation to these structures. ${ }^{3}$ Despite being benign, the recurrence rate after resection is high- hence the name. ${ }^{4}$

Sonography is inconclusive; shows a mass that is hypoechoic or appears cystic. ${ }^{3}$ On CT it appears hypodense relative to muscles with a layering or swirled pattern after contrast administration. ${ }^{4}$ MRI shows the classical high intensity on $\mathrm{T}^{2}$-weighted images and a swirled appearance. ${ }^{3}$

Case reports have shown increased growth of the tumor during pregnancy and resolution postpartum, thus suggesting a hormonal dependency as suggested by estrogen receptor and progesterone receptor (PR) positivity. ${ }^{5,2}$

\section{Conclusion}

The importance of this case is the knowledge of this lesion. This diagnosis should be considered in any young woman with a well-defined mass arising from the perineum even if the lesion appears to be fluid containing on ultrasound. Since interval growth is very slow, both surgical and conservative management can be offered to the patient.

\section{References}

1. Venkateswar R. Surabhi NGF. Aggressive Angiomyxomas:A Comprehensive Imaging Review With Clinical and Histopathologic Correlation. AJR. June 2014;202:1171-1178.

2. Kamal Malukani AVVDCaSD. Aggressive angiomyxoma in pregnancy: A rare and commonly misdiagnosed entity. J Lab Physicians. Apr-Jun 2018;10(2):245-247. 
3. Eric K. Outwater BEMBJWESS. Aggressive Angiomyxoma: Findings on CT and MR imaging. AJR. 1999;172:435-438.

4. Rashid Saif Al-Umairi AKFMAB. Aggressive Angiomyxoma of the Pelvis and Perineum: A Case Report and Literature Review. Oman Med J. Nov 2016;31(6):456-458.
5. Orfanelli T KCVSVG,MN. A Case Report of Aggressive Angiomyxoma in Pregnancy: Do Hormones Play a Role? Case Rep Obstet Gynecol. Nov 2016;Article ID 6810368:4. 